\title{
LAS TENDENCIAS DE LA INVESTIGACION EN ENFERMERIA
}

Margarita Velasco A. *

Vengo de un país llamado Ecuador, localizado al otro lado de la América Latina, de diez milliones de habitantes, con población indígena o con ancestros indios, en su gran mayoría. Enclavado en la mitad del mundo y que, junto a Venezuela, Colombia, Perú, Bollvia y Chile, está situado a lo largo de los Andes, en la región en la que, a decir del Banco Iteramericano de Desarrollo, se concentran los más ricos recursos naturales del subcontinente (2).

Traigo el saludo y la solidaridad de las compañeras y companeros enfermeros ecuatorianos quienes nos unimos a la celebración de los 50 años de la Escuela de Enfermerla de la Universidad de São Paulo. Celebración de los 50 años de la Escuela de Enfermería de la Universidad de São Paulo. Celebración que coincide con el primer medio siglo de la Escuela Nacional de Enfermería de Ecuador, institución a la que me pertenezco.

Este hecho, recuerda el pasado común que tiene el desarrollo de la educación de enfermería en América Latina, señalado por Alina Maria de Almeida SOUZA (13), y en el que la presencia, en la segunda guerra mundial (1942), de organizaciones internacionales, como la fundación Rockefeller, trajo a enfermera norteamericanas a nuetros países para que organizaran el sistema educativo de enfermería.

Estos hechos son testimonios históricos de que en América Latina, y en la enfermería lationamericana, los determinantes político-económicos y sociales han tejido una gruesa red de interrelaciones, que nos permiten hoy, a finales de siglo, y cuando también se cumplen 500 años de opresion europea sobre lo que ellos denominaron América; sentranos a hablar de lo que tenemos en comun, que posiblemente es más de lo que tenemos de diferentes. Porque los pueblos, como los nuestros, que tienen la misma dependencia y causas de la miseria y la desigualdad, están hoy ante el mismo desafio y que consite, como lo dice Carlos FUENTES (4) en transformarnos de POBLACION, a menudo pasiva, sometida, explotada, manipulada, a CIUDADANIA activa, liberándose, autónoma frente al principe y al gerente.

\section{El contexto}

La investigación en enfermería, al igual que la investigación científica debe responder a las necesidades del contexto social, economico, cultural, y al

- Enfermera. Subdirectora de la Escuela de Salud Pública; profesora de Política Social de las Maestrias de Administración e Investigación y Salud Ocupacional de la misma escuela. Docente de la Escuela Nacional de Enfermerfa de la Facultad de Ciencias Médicas de la Universidad Central del Ecuador. 
momento histórico por el que atraviesan nuestros países. De lo contrario, no se explica la razom de ser de la ciencia, que es el bienestar de la humanidad.

Por tanto, las tendencias de la investigacón en enfermería deben ser analizadas a la luz de su correspondencia con la realidad del contexto para poder emitir criterios respecto a su papel social como profesión. Esta afirmación la hacemos ya que la investigación permite interpretar, medir, evaluar el quehacer de un ámbito específico, en este caso, de salud, y en él, enfermería. Por eso partiremos de una revisión global de nuestra circunstancia como latinoamericanos, para luego obervar las tendencias de investigacion en algunos países de América Latina, y explícitamente, en Ecuador.

La situación de la América Latina en la década del 90, es el resultado la llamada "crisis de la deuda" que se origina en los años ochentas. Los problemas financieros que este hecho provocó y el ajuste que impuso sobre los paíeses más fuertemente endeudados, determinaron que los niveles de inversión se redujeran en términos absolutos y lo que es más grave aún, que los niveles de vida de la población de la reigión sufrieram la mayor caída experimentada en medio siglo (2).

Según los organismos financiadores (BID, Banco Mundial), América Latina deberá resolver los siguientes problemas en los años 90:

- El logro de una adecuada y sostenida tasa de crecimiento global

- La reducción de la incidencia de la pobreza absoluta y las disparidadess de ingressos.

- La conservación de los recursos naturales y el mejoramiento de la calidad ambiental

- La reducción del tamaño del Estado, a través de reformas, y el impulsoo a la privatización (2).

Dicha reducción del tamaño del Estado aparece como un problema grave, ya que dada la crisis, más necesaria se deberia volver la intervención estatal para atender los problemas sociales decurrentes de ella. La reducción y privatización del Estado se plantea porque al haberse interrumpido el crecimiento sostenido de la economía, la crisis reduce no solo los recursos de los diferentes sectores sociales, sino también los que el sector público requiere para viabilizar el funcionamiento de sus instituciones.

La crisis económica pone de manifiesto la insuficiencia estructural de la región para sostener ritmos de crecimento y la incapacidad para dar respuestas al problema del subdesarrollo.

Hoy, en vez de que los países industrializados transfieran recursos que alienten la formación de capital en América Latina y el Caribe, ellos absorben ahorros en gran escala. Durante 1987, más de US. 28.000 millones fueron tranferidos fuera de nuestros países y durante los últimos 5 años, los fondos perdidos, sobre todo por concepto del pago de la deuda externa acumulada, llegó a alcanzar la cifra de 130.000 millones de dólares (8).

Frente a la crisis provocada por la crisis a su vez, del modelo económico, agravado por el endeudamiento externo, y la situación económica mundial, los países defenieron políticas de ajuste para enfrentarlas. En lo social ello provocó incremento de la desocupación y la incapacidad de compra de grandes sectores de la población, y problemas en los servicios básicos como salud, vivienda, educación y transporte. 
Desde un punto de vista político, los países de la región se enfrentan hoy a la dif́cil situación de asegurar la gobernalidad de sus sociedades, al tiempo de resolver la situación económica. El fracaso de los autoritarismos de viejo y nuevo cuno en su empeño por resolver los desafios de la crisis, unido a la movilización de las fuerzas democráticas, han abierto en América Latina, una oportunidad histórica para la definitiva consolidación de la democracia.

En cuanto a salud, el impacto de la crisis provoca que grandes sectores de la población, de la mayoría de nuestros países sigan sin tener acceso real a los servicios de salud.

Un estudio especial, realizado por la OPS constató que el gasto real per cápita efectuado por los Ministerios de Salud Pública de 10 paises de la América Latina, en la década del ochenta, aumentó sólo en 4 de ellos, y en seis se constató su disminución. No es fortuito que ante semejante cuadro muchos especialistas hayan comenzado a hablar de la DEUDA SANITARIA que el Estado ha contraído con la población, sobretudo la más pobre (9).

Las consecuencias de esta deuda sanitaria se las observa en la elevación de las tasas de mortalidad infantil, en el aparecimento de enfermedades que antes no existian (como el cólera), en la limitación de inversiones de capital para áreas fundamentais como saneamiento básico, mantenimiento y conservación de los equipos y plantas físicas de los estabelecimentos de salud.

Así mismo, se constata una limitación para gastos corrientes, afectando así el normal funcionamiento de programas para la atención de problemas prevalentes en la región. O, por otro lado, concentrando recursos en programas de acción, orientados a la atención de problemas prioritarios y de mayor impacto inmediato como es el caso de los programas de inmunizaciones, supervivencia infantil o subsidios alimentarios (8).

Las medidas de ajuste en el plano de la salud obedecen a políticas regionales entre las que la focalización de la atención de salud debe ser mencionada.

En países como los nuestros, en los cuales los límites entre pobreza, y miseria son subjetivos, y en los cuales el grado de empobrecimento de amplios sectores de la población se la constata a simple vista, o por el grado de violencia social e inseguridad de las principales ciudades de la region, se prentende que la atención de salud tenga una nueva connotación en lo que se refiere al principio de equidad de la doctrina sanitaria. En efecto, se habla de atender hoy solo a los más pobres de entre los pobres, con acciones de salud que logren salvar sus vidas, aunque no tengan éstos acceso, ni a empleo y por consiguiente, a alimentación segura, peor aún a educación y a recreación.

Finalmente las consecuencias cotidianas a las que nos enfrentamos son que la crisis económica ha arrojado de sus hogares, de sus escuelas, a los nifios que antes podían tener acceso a la educacion. Ahora deben ir a las calles a limpiar parabrisas, hacer peripecias para ganar dinero, unirse a bandas criminales, o ser asesinados por bandas esquizoides.

Pero además de la crisis, este fin de siglo, nos ha preparado una serie de sorpresas de las que lentamente comenzamos a salir. Nos ha dejado una lección a rescatar y es que todo cuanto parecia muerto - religiones, regiones, memorias, , lenguas, creencias, magias, sueños - estaba vivo, coincidiendo con todo aquello) 
que puede oler a modernidad: desde un fax hasta una fibra optica, un jet o un teléfono celular.

Parte de la respuesta que la investigación debe dar, es cómo, nosotros, ciudadanos de esta región que vive la fructifera tensión entre opuestos. Entre lo antiguo y lo nuevo, entre lo tradicional y lo moderno. Cómo vamos a vivir esta modernidad tecnologica con la presencia cultural que reúne, por definición pasado y porvenir en el presente? Como vamos enfrentar la crisis que, en lo particular de salud, nos atane a todos los trabajadores de la salud y no solo a una profesion?

\section{Las tendencias de investigacion en enfermería en los ochentas}

En el primer Coloquio Panamericano de Investigación en Enfermería, Nelly GARZON (5), Presidenta del Consejo Internacional de Enfermeras CIE, afirmaba que la investigación de enfermería debe responder a las variables del contexto social actual y futuro, y contribuir a resolver las necesidades globales de salud de la sociedad y a mejorar la calidad de vida.

Decía, además, que la investigación se orienta a producir e encrementar el conocimiento de la práctica de enfermería, de las especificaciones de la misma, del espacio que tiene en la práctica de salud en general. Nosotros añadimos, y de las determinaciones que sobre dicha práctica ejerce la sociedad, el Estado y el desarrollo histórico, económico, social, cultural y político de los diferentes países.

La producción científica de enfermería sistematizada y periódica está directamente relacionada con la formación de los recursos humanos de enfermería a nivel de pre y postgrado. Citaremos dos ejemplos. En el Brasil, 208 tesis de enfermería (de postgrado), fueron catalogadas por el Centro de Estudios e Investigaciones en Enfermería entre 1979 e 1983 (1).

En el Ecuador (país en el que el nivel de postgrado es de limitado acceso para enfermeras, no existiendo sino desde 1991 un postgrado en enfermería pediátrica), desde 1965 se encuentran registradas 609 trabajos de tesis de estudiantes del pre-grado, en 5 de las 11 escuelas de enfermería que existem en el país (10). El número de trabajos de investigación realizados por personal de enfermería en su ejercicio professional no rebasa los 60 en el mismo espacio de tiempo (14).

Alina María de Almeida SOUZA (12), corrobora lo anterior, cuando afirma que la producción de investigaciones en enfermería aparece de forma más regular a partir de que la profesión se incerta en la universidad en la década del sesenta.

En el caso de Ecuador, la produción está directamente relacionada con el aparecimento, en 1965, del nível de licenciatura, que es el título que actualmente se otorga luego de cuatro años de estudios. (Además de éste, los niveles de enfermería existentt:s son: enfermeras generales con tres años y los auxiliares de enfermería con cursos de 9 meses).

El estudio comparativo sobre Las tendencias de investigación en América Latina, patrocinado por la OPS-OMS y realizado en 1988 (3), permitió por vez primera identificar las áreas temáticas de privilegio de la investigacion de enfermería en el período 1983-87. Resaltamos en el siguiente cuadro, los resultados encontrado en 4 países. 
TENDENCIAS DE INVESTIGACION EN ENFERMERÍA EN

CUATRO PAISES DE AMERICA LATINA

\begin{tabular}{|c|c|c|c|c|}
\hline PAISES/TEMAS & COLOMBIA & EQUADOR & MEXICO & BRASIL \\
\hline $\begin{array}{l}\text { Asistencia de } \\
\text { Enfermeria (Diag. } \\
\text { Trat. Prom. Prev.) }\end{array}$ & 54.6 & 49.0 & 45.98 & 49.7 \\
\hline $\begin{array}{l}\text { Administración } \\
\text { Formación de R.H. }\end{array}$ & 17.6 & 37.0 & 43.51 & 27.2 \\
\hline $\begin{array}{l}\text { Factores que } \\
\text { influyen en la } \\
\text { práctica }\end{array}$ & 27.2 & 14.0 & 9.6 & 23.1 \\
\hline
\end{tabular}

\begin{tabular}{lllll}
\hline TOTAL & $100 \%$ & $100 \%$ & $100 \%$ & $100 \%$
\end{tabular}

FUENTE: MEMORIAS DEL 1er COLOQUIO PANAMERICANO DE INVESTIGACION EN ENFERMERLA ACOIFAN, OPS/OMS, 1989

Elaboracion: Margarita Velasco

Esta tabla nos permite evidenciar lo siguiente:

- Una marcada tendencia a investigar temas que giran en torno a la asistencia de enfermería, tales como diagnóstico, tratamiento, promoción y prevención, con un claro ènfasis alrededor de los individuos, el hospital, y la atención curativa de enfermería. Los porcentajes más bien son uniformes para los cuatro países, ubicándose entre el 46 y $54 \%$ de los trabajos que se consideraron en la muestra.

- La temática sobre administración y formación de los recursos humanos es abordada de manera heterogénea entre los 4 países. México llega al $43 \%$, Ecuador al $37 \%$, Brasil al $27,2 \%$ y Colombia al $17,6 \%$.

Para avanzar en el análisis, nos referiremos a lo que específicamente sucede en el Ecuador. Los estudios de administración abordan la problemática de las salas de hospitales, pero centradas en lo que sucede con el personal de enfermeria. Sin embargo, la explicacion que permita profundizar en las interrelaciones y determinantes de estos problemas, no se circunscriben a dicho personal sino a lo institucional, en el que existen otros actores, oteas fuerzas, y para transformarlas, no se puede contar solo con lo que enfermería piensa hacer porque no termina por incidir en la esencia del conflicto. Además, al describir el contexto de la crisis, los hospitales, son las instituciones que mas duramente fueron golpeadas con las reducciones de recursos económicos. No se rebase el ámbito específico, como reiterando que le mundo comienza y termina en lo de enfermería, como que hubiera un temos a ser parte del mundi, y asumirse como actor.

- Factores que influyen en la práctica es otro de los temas con desarollo heterogéneo entre los 4 países. En este módulo se incluye: el marco legal de la práctica de enfermería, las políticas de salud y enfermería, la planificación y desempeño político. México es el que menor número de trabajos posee en este tema (9.6\%), seguido de Ecuador (14.5\%). Brasil, con el $23 \%$, se acerca al $27 \%$ de Colombia. 
En base a lo observado en el Ecuador, comentamos que el análisis de la relación entre determinantes del contexto y práctica de enfermería, que permiten una comprensión profunda de la misma, recién aparece como enfoque en los afios 80. Esto es el resultante de: la influencia de los grupos que se forman en torno a la medicina social, sobretodo en el Centro de Estudios y Asesoría en Salud (CEAS), del apoyo que Juan César García presta, desde la OPS, a la investigación de enfermería, de la formación en áreas sociales de algunas pocas investigadoras, y de la presencia de egresadas del curso de especialización en Investigación y Administración en Salud, hoy convertido en la Escuela de Salud Pública.

El estudio de la OPS suministra otra información valiosa, que no la tomaremos, por no considerarlos pertinentes para el tema que abordamos.

De 1988 a 1992, no tenemos datos sobre lo que ocurrio en los palses que antes hicimos referencia, para compararlos. Como no hemos tenido acceso a datos globales de la región, centraremos el análisis a lo que sucedió en el Ecuador.

\section{Tendencias de investigación en enfermería en el Ecuador 1988-1992}

Los estudios sobre asistencia de enfermería fueron temas abordados en los trabajos de investigación del pre-grado. Una muestra de ello es que de 177 tesis de grado de estudiantes de las últimas promociones de licentiatura de las Esculeas de Enfermería más importantes de Ecuador Facultades de Enfermería del país, el $38.8 \%$ corresponden a esta área*.

Por primera vez aparece incluída en esta temática, investigaciones referidas a desarrollo de tecnología en la atención de enfermería.

Sin embargo, llama la atención el incremento de estudios epidemiológicos y de diagnóstico comunitario que aparecen (33.8\%), junto a trabajos que comienzan a abordar un tema nuevo y de vital importancia para un país multiétnico y multicultural como el Ecuador, y que es el de cultura y salud, trabajada por el $5,6 \%$ de tesis.

Este hecho se puede explicar porque a partir de 1988 aparecen grupos que plantean como necesidad de la política de salud el desarrollar el nível primario de atención a través de propuestas innovadoras. A ello se une el avance del movimiento indio que aparece en la escena pública como uno de los grupos de la sociedad civil con mayores posibilidades de intervención, coherencia y movilización de grupos. Se rescata, por tanto el hecho del país pluricultural, y ello necesariamente toca a la salud.

Estudios sobre administración, el $28.2 \%$., se encontraron sobre todo en la Escuela Nacional de Enfermería, aplicando el enfoque estratégico, y mediante la participación del personal de los servicios. Educación fue un tema no estudiado.

Con respecto a las enfermeras que trabajan en las instituciones de salud hospitalarias y de atención primaria, se planteó y se inició una investigación sobre la Calidad de la atención de enfermería de acuerdo a la percepción que la comunidad tiene.

- PUCE. Facultad de Enfermena. Centro de Documentacion. Archivo, 1992 y BAJAÑA, Susana. Profesora de la Cátedra de Investigación. Entrevista personal. Quito, octubre, 1992. 
La situación laboral y su repercusión en la salud del personal de enfermería es un tema que comienza a aparecer continuamente en estos últimos tres años.

Un hecho a destacarse es el que aparecen nuevos grupos de investigadores en enfermería, formados por enfermeras de hospitales, o promocionados por primera vez por instituciones que años antes no fueron protagónicas en el impulso a la investigación de enfermería, tales como la Federación Ecuatoriana de Enfermeras-os, la División Nacional de Enfermería del Ministerio de Salud Pública y grupos multiinstitucionales de enfermeria*.

Se observó una ausencia de investigaciones por parte de las Escuelas de Enfermería del país como cuerpo docente, circunscribiendo su papel en la producción del conocimiento científico, a la dirección de tesis de sus estudiantes**. En docencia, en algunas escuelas de enfermería, por ejemplo, la Escuela Nacional, con un curriculum comunitario desde 1981, y un grupo de profesores que intenta incorporar la metodología problematizadora al proceso educativo, se densencadeo una discusión teórica alrededor de la investigación tradicional y la investigación participativa, que llevó a serias dudas respecto a la formación de la estudiante como investigadora. Las perguntas del debate eran: para qué investigar? y por qué no manejar la investigación cualitativa frente al dominio de instrumentos de la investigación tradicional? Cómo operacionalizar la investigación participativa, si el proceso educativo tiene como limitantes fundamentales el hecho de que los espacios de tiempo escolares, impiden el acompañamento de los procesos de cambio en los servicios y en la comunidad que siempre son demasiado lentos frente a la exigencia de la universidad en el cumplimiento de los programas?

\section{Los problemas para la realización de investigaciones}

Los principales problemas que enfrentó el personal de enfermería en el Ecuador para poder realizar trabajos de investigación en estos últimos años fueram:

- Ausencia absoluta o limitadísimo acceso a recursos económicos para poder realizar sus investigaciones o para finalizarlas, ello impidió la toma de ciertos datos complementarios en las investigaciones ya iniciadas, o el uso de la informática, teniendo que realizar en muchos casos estudios nacionales, con procesamientos manuales de los datos***.

El proceso de investigación que depende, en el caso de la Universidad, de la financiación que ofrece el Consejo de Universidades y Escuelas

- gallardo, N. Ex-Directora de la División Nacional de Enfermería del MBP. Entrevista personal. Quito, octubre, 1992.

** VELASCO, Maria de Lourdes. Secretaria Ejecutiva de ALADEFE, Jefe de Cátedra de Investigación. Entrevista personal. Quito, octubre de 1992.

** BEGOVIA, Rocio. Coordinadora de la Comisión Cientifica de la FEDE Entrevista personal. Quito, octubre, 1992. 
Politécnicas, a criterio de algunas de nuestras encuestadas, se ha burocratizado, centralizado, exigiendo requisitos que no pueden ser llenador por las enfermeras ecuatorianas, volviendo a los fondos inaccesibles*.

- El tiempo para investigar no existe para el personal de los servicios. Las pocas investigaciones que se realizaron fueron en tiempo extra-trabajo porque las instituciones de servicio de donde provienen los nuevos grupos de investigadoras, no liberaron a éstas de sus jornadas de trabajo, a pesar de que las mismas realizaban estudios sobre salud de las enfermeras y condiciones de trabajo**.

- La difusíon escrita en estos áltimos años se volvió casi imposible para la producción de enfermería, limitándose a resúmenes escuetros en ciertas revistas de la profesión, de circulación limitada.

- En cuanto al manejo del método cientifico, los problemas más frecuentemente encontrados se refirieron al uso de la estadística en los estudios realizados.

Si bien una de las debilidades detectadas en el estudio de la OPS - OMS realizado em 1988 para el caso de Ecuador, fue la ausencia de políticas de investigación en Enfermería, en ese sentido se logró avanzar. La Asociasión Ecuatoriana de Escuelas y Facultades de Enfermería de Ecuador, logro definir los lineamientos generales que guiarán la formulación de las políticas de investigación de enfermería. En ella se priorizan las siguientes áreas:

- Salud del personal de enfermería y condiciones de trabajo

- Administración de enfermería

- Educación

- Desarrollo tecnológico en enfermería

- Marco legal de la práctica de enfermería (11).

\section{5 . Reflexion final}

La descripción que ha precedido a esta parte, ha permitido observar una débil relación entre los temas investigados y el contexto, tal parece que la crisis impide pensar en el futuro, o que el presente se ha convertido en un continum, que no nos conduce a ningún lugar.

- PUCE. Facultad de Enfermería. Centro de Documentación. Archivo, 1992 y BAJAÑA, Susana. Profesora de la Cátedra de Investigación. Entrevista personal. Quito, octubre, 1992. ** BEGOVIA, Rocio. Coordinadora de la Comisión Cientifica de la FEDE. Entrevista personal. Quito,
octubre, 1992 . 
Está claro, al menos para las enfermeras-os ecuatorianas, que la enfermería es una práctica social. Pero, frente a los planteamientos del neoliberalismo, a la privatización para construir empresas de salud, y al enanismo del Estado, cómo construir el ejercicio de la práctica social desde enfermería?.

No tenemos las respuestas perfectas, porque nos han cambiado las preguntas, por eso tan solo esbozare algunos planteamientos.

1. La hegemonía médica fue construída en base a un poder tradicional dado por la capacidad, proveniente de la división del trabajo, de diagnosticar y recetar. Ese poder es técnico y es económico, porque el médico es quien al efectivizar el acto terapético, realiza el valor de la mercancía (6).

A diferencia del médico, la enfermera no tiene un poder tradicional. Su objeto de trabajo es a veces definido como el cuidado de los individuos. Hoy podríamos decir que es el cuidado del cuerpo social. El médico receta, la enfermera cuida. El primero comanda el proceso de trabajo para obtener um producto. Ello es claro sobretodo en el ámbito hospitalario, en el que los roles y los espacios de cada profesión están perfectamente marcados, y nadie sobrepasa los límites normatizados. Cuando se cambia el escenario, cuando éste es la comunidad, y en ella no están pacientes, sino ciudadanos que participan, y los problemas rebasan lo biologico-curativo, se necesita del trabajo colectivo para enfrentar los problemas del colectivo. Los miembros del equipo de salud se complementan y no temen cubrir los espacios de los otros, porque la realidad envuelve y demanda soluciones inmediatas. El médico continúa responsabilizandose de la morbilidad. La enfermera amplia su ámbito, hacia la relación con la población, hacia el trabajo en la población y comienza a generar y a acumular un nuevo poder. El que emana del ejercicio de sua politicidad.

2. Lo político, que tiene doble sentido, en cuanto posibilidad de acumular poder, y de impregnar de una direccionalidad todos los actos, fortalecen el ejercicio de enfermería como práctica social. Ese poder político de la enfermera radica hoy, en la posibilidad de cohesionar al personal de enfermeria. En tiempos de individualismo, no hay nada más subversivo como plantear la unión. La unión de enfermería con la población y con el resto de trabajadores de la salud (7).

Cada uno de estos puntos de unión tienen ámbitos de responsabilidad específica y común en la organización de enfermería. La unión de las enfermeras-os, es la tarea fundamental de los gremios y de todos sus asociados. La unión com la población es en el trabajo en la comunidad, y en el hospital que tiene una concepción de institución abierta a una red de servicios y a una población. La unión con el resto de trabajadores de la salud, es parte del trabajo diario, por la consecusión de objetivos comunes. 
3. La construcción de ese poder de enfermería tiene una direccionalidad, si es que se busca como práctica social. Esto significa el desarrollo de un pensamiento estratégico, que considere el pasado, pero que permanentemente mire al futuro, para construirlo desde el presente.

El futuro es el proyecto historico de sociedad que debe ser factible y visible desde el hoy. Una sociedad democrática, pluralista, justa, feliz. No es ésta la eterna búsqueda de lo humano?.

Cómo llegar a ello? Cómo instrumentar lo teórico, lo metodológico y lo político?.

Una vía puede ser, desarrollando um pensamiento estratégico que vaya concretizando, contextualizando, operacionalizando, y permanentemente analizando las posibilidades de viabilidad y factibilidad de los objetivos sociales, desde lo especifico de enfermería.

Por tanto, la investigación que se propone impulsar debe ser parte del camino hacia la utopia, en la que tanto necesitamos sostenernos los lationoamericanos, porque sin suenos, los hombres y mujeres podemos comenzar a morir. La búsqueda del conocimiento científico a través de la investigación, dentro de ese marco estratégico, será para alimentar la reflexión que precide y precede a la acción, no hacia el utilitarismo de la vida cotidiana, sino hacia la construcción del sujeto social (7).

4. Esa investigación-militante que visualizamos, unida a la construcción del futuro en el presente, es la correspondencia entre la práctica a desarrollarse con el conocimiento a producirse, entre la necesidad de transformación con el requerimiento filos6fico (7).

Conocer, no para desarrollar el cientifismo que nos satisface individualistamente; tampoco para denunciar y sentir catársi. Conocer porque hoy es el tiempo de actuar.

El ejercicio de la ciudadanía pasa por el fortalecimiento de la sociedad civil, y ello significa participar de la vida del país, buscar soluciones, pornelas en práctica, acumular fuerzas y no dejar le luchar porque los sueños sean la realidad.

De esa forma, la investigación no solamente debe servir para buscar la GENESIS del problema, sino al mismo tiempo, para responder al requerimiento de comprender las OPCIONES que se pueden seguir para engendrar acciones políticamente dirigidas hacia objetivos estratégicos (7).

Las enfermeras, mayoritariamente, mujeres, siempre demonstraron un coraje especial, probablamente porque su práctica es social, está cerca y con la gente. Investigaron, incluso sin tener dinero ni tiempo para hacerlo. Hoy están preocupadas de sus países, de sus pueblos. Su triple carga de mujeres, madres y trabajadoras nunca fue freno. El espacio nuevo que la atención primaria de salud créo para ampliar su papel, para entregarle a ella el vínculo con la población, para fortalecer el trabajo en equipo multidisciplinario, para abrir los lugares demandados por la población en el control y gestión de los servicios de salud, la encuentra comenzando su madurez como profesión. Sin 
inseguridades sobre los que es su quehacer específico, más comprometida, más involucrada con el cuerpo social, al que ha comenzado a cuidar, de la manera menos pasiva y subordinada posible.

\section{REFERENCIAS BIBLIOGRÁFICAS}

1. ALMEIDA, M. C. P. de; YAZLE, J. E. R. O saber de enfermagem e sua dimensão prática. São Paulo, Cortez, 1986.

2. BANCO INTERAMERICANO DE DESARROLLO. Processo económico y social en América Latina: Informe 1991. Washington, 1991.

3. COLÓQUIO PANAMERICANO DE INVESTIGACIÓN EN ENFERMERIÁ, 1, BOGOTÁ, 1991. Memórias. Bogotá, ACOFAEN, s.d.

4. FUENTES, C. Reflexiones de fin de siglo. El Comercio, Quito, 11 oct. 1992.

5. GARZON, N. Investigación en enfermería: orientaciones internacionales, In: COLÓQUIO PANAMERICANO DE INVESTIGACIÓN EN ENFERMERÍA, 1, Bogotá, 1991. Memórias. Bogotá, ACOFAEN, s.d.

6. GRANDA, E. Acerca de la propuesta de introducir el termino praxis. Quito, Escuela de Salud Pública, 1992.

7. La planificación y la educación de enfermería. /Ponencia para el Congreso por el Cincuentenario de la Fundación de la ENE, Quito, octubre, 1992.

8. OZLAK, O .et al. Descentralización de los sistemas de salud: el estado y la salud. In: Paganini, J.; Capote-Mir, R. Los sistemas locales de salud: conceptos, métodos, experiencias. Washington, Organización Panamericana de la Salud, 1990, p. 59-81.

9. PAGANINI, J. M.; CAPOTE-MIR, R. Los sistemas locales de salud: conceptos, métodos, experiencias. Washington, Organización Panamericana de la Salud, 1990 (Publicación cientifica, 519).

10. RAMIREZ DE ROMERO, L. Experiencias de investigación en enfermeria en la Asociacion Ecuatoriana de Escuelas y Faculdades de Enfermería. In: SEMINARIO TALLER SOBRE POLITICAS Y LINEAS DE INVESTIGACIÓN EN ENFERMERIA, Quito, 1991. Memórias. Quito, ABEDEFE, 1991.

11. SEMINÁRIO TALLLER SOBRE POLITICAS Y LINEAS DE INVESTIGACIÓN EN ENFERMERIA, Quito, 1991. Memórias. Quito, ABEDEFE, 1991.

12. SOUZA, A. M. A de. A enfermagem: análise das principais pesquisas e seus problemas. In: ORGANIZAÇÀO PAN-AMERICANA DE SAÚDE. As ciências sociais em saúde na América Latina: tendencias e perspectivas. Washington, 1985.

13. . Desenvolvimento dos senviços de assessoria de enfermagem na Organizaçào Pan-Americana da Saúde: impacto na educaçăo de enfermagem latino-americana (1940-1980). Washington, 1982.

14. VELASCO, M. La participación de enfermería en investigaciones en salud. Rev. Fac. Cienc. Méd, Quito, p., mar. 1985. 